\title{
The Effects of a Subpsychotic Dose of Ketamine on Recognition and Source Memory for Agency: Implications for Pharmacological Modelling of Core Symptoms of Schizophrenia
}

\author{
Garry D Honey', Chris O’Loughlin', Danielle C Turner', Edith Pomarol-Clotet', Philip R Corlett' \\ and Paul C Fletcher*,I
}

'Department of Psychiatry, University of Cambridge, Cambridge, UK

\begin{abstract}
Ketamine is increasingly used to model the cognitive deficits and symptoms of schizophrenia. We investigated the extent to which ketamine administration in healthy volunteers reproduces the deficits in episodic recognition memory and agency source monitoring reported in schizophrenia. Intravenous infusions of placebo or $100 \mathrm{ng} / \mathrm{ml}$ ketamine were administered to 12 healthy volunteers in a double-blind, placebo-controlled, randomized, within-subjects study. In response to presented words, the subject or experimenter performed a deep or shallow encoding task, providing a 2 (drug) $\times 2$ (depth of processing) $\times 2$ (agency) factorial design. At test, subjects discriminated old/new words, and recalled the sources (task and agent). Data were analyzed using multinomial modelling to identify item recognition, source memory for agency and task, and guessing biases. Under ketamine, item recognition and cued recall of deeply encoded items were impaired, replicating previous findings. In contrast to schizophrenia, there was a reduced tendency to externalize agency source guessing biases under ketamine. While the recognition memory deficit observed with ketamine is consistent with previous work and with schizophrenia, the changes in source memory differ from those reported in schizophrenic patients. This difference may account for the pattern of psychopathology induced by ketamine.

Neuropsychopharmacology (2006) 31, 4I3-423. doi: I 0. 1038/sj.npp. I 300846; published online 3 August 2005
\end{abstract}

Keywords: ketamine; schizophrenia; source memory; multinomial modelling; NMDA

\section{INTRODUCTION}

The ability to distinguish internally from externally generated thoughts and actions is fundamental to establishing and maintaining a representation of oneself. A failure in this ability is considered central to some of the symptoms of schizophrenia, including auditory hallucinations, passivity phenomena, thought insertion, and delusions of control (Frith, 1987, 1992; Frith and Done, 1989). The inability to recognize one's own thought processes may result in a tendency to form anomalous external attributions, which has been termed 'autonoetic agnosia' (Keefe, 1998). Such deficits have been demonstrated in schizophrenia using source memory (Brebion et al, 1999, 2000, 2002; Danion et al, 1999; Keefe et al, 1999, 2002; Moritz et al, 2003; Morrison and Haddock, 1997; Stirling et al, 1997;

\footnotetext{
*Correspondence: Dr PC Fletcher, Department of Psychiatry, University of Cambridge, Addenbrooke's Hospital, Brain Mapping Unit, Cambridge CB2 2QQ, UK, Tel: + 441223 336988, Fax: + 441223 33658I, E-mail: pcf22@cam.ac.uk

Received 5 April 2005; revised 9 June 2005; accepted 17 June 2005 Online publication: 22 June 2005 at http://www.acnp.org/citations/ Npp06220505022 I/default.pdf
}

Vinogradov et al, 1997) and reality monitoring paradigms (Bentall et al, 1991; Brebion et al, 1996, 1997; Harvey, 1985; Keefe et al, 2002; Moritz et al, 2003). Source memory refers to an individual's ability to recollect and use contextual information. Reality monitoring, a specific example of source memory, describes the discrimination of self- from externally-generated information (Johnson and Raye, 1981). In such tasks, people with schizophrenia demonstrate the tendency to externalize information generated internally (Bentall et al, 1991; Keefe et al, 1999, 2002; Moritz et al, 2003) and show greater confidence in these erroneous source judgements (Moritz et al, 2003).

Ketamine, an NMDA receptor antagonist, has increasingly been used as a human pharmacological model of schizophrenia, based on the NMDA receptor hypofunction model of the illness (Krystal et al, 1994; Olney and Farber, 1995; Tamminga et al, 1995). Administration of ketamine in healthy volunteers induces psychopathology, as rated by the Brief Psychiatric Rating Scale (BPRS), which include experiences associated with autonoetic agonosia, such as delusional ideation (Krystal et al, 1994; Duncan et al, 2001; Malhotra et al, 1997; Newcomer et al, 1999). 
Relevant to the view that episodic memory represents a core cognitive deficit in patients with schizophrenia, episodic memory impairment has been consistently observed following ketamine administration in healthy volunteers (Ghoneim et al, 1985; Harris et al, 1975; Hetem et al, 2000; Krystal et al, 1994; Malhotra et al, 1996; Newcomer et al, 1999; Radant et al, 1998). We have previously demonstrated that ketamine, when administered prior to the encoding of information, disrupts both recognition and source memory (Honey et al, in press), consistent with previous findings of (Morgan et al, 2004a). The disruption we previously observed in source memory was manifest as an increased tendency to bias erroneous responses towards the task producing the weakest encoding. The aim of the current study was further to explore the impact of ketamine on memory, using a task that emphasizes a particular form of source monitoring: agency source monitoring, that is, recalling whether information was self- or externally-generated. This form of source monitoring may be of particular relevance to schizophrenia, which is associated with difficulties in distinguishing between and remembering information generated from internal and external sources (Keefe et al, 1999, 2002), and may be particularly related to positive symptoms (Brebion et $a l, 2002$ ) and their response to treatment (Keefe et al, 2003).

This study explored the impact of ketamine on source memory. We sought primarily to determine whether ketamine infusion has differing effects upon memory for whether words were externally or internally generated. Our goal was to specify the nature of ketamine-induced changes on this form of source monitoring and to relate these to deficits found in schizophrenia with a view to evaluating further the NMDA receptor hypofunction model of the disease. On the basis of deficits reported in schizophrenia, we predicted that ketamine administration would lead to a failure of monitoring of self-generated thought, reflected in an increased bias towards reporting external representations of internally generated information.

One important consideration for this task is that internally generated information shows a mnemonic advantage: the 'generation-effect' (Slamecka and Graf, 1978). We attempted to account for this by using a second source manipulation, comparing deeply encoded information (semantic association) with a shallow encoding condition (word stem completion) which did not differ in internal/external agency. Thus, we used a $2 \times 2$ factorial design in which two depths of encoding (deep $v s$ shallow) were crossed with two types of generation (internal vs external). The analytical method of choice in such a task is multinomial modelling (Batchelder and Riefer, 1990), an approach reported in our previous study on the effects of ketamine on source monitoring (Honey et al, in press), and also employed in previous investigations of self-monitoring deficits in schizophrenia (Keefe et al, 1999, 2002).

One further aspect of our design is central to the research question. The dose of ketamine was selected on the basis of our previous work indicating that cognitive deficits are evident at $100 \mathrm{ng} / \mathrm{ml}$, while the psychotogenic effects are extremely mild at this dose (Honey et al, in press; Honey et al, 2003). The interpretation of psychopharmacological effects on specific cognitive processes are thereby inter- pretable largely in the absence of confounding effects of concomitant psychopathology. This separation of cognitive and psychotic effects of the drug exploits a key advantage afforded by pharmacological modelling of disease processes.

\section{METHODS}

\section{Subjects}

In all, 13 right-handed healthy volunteers (eight males) were tested with their informed consent. The study was approved by the Addenbrookes NHS Trust research ethics committee and was carried out in accordance with The Code of Ethics of the World Medical Association (Declaration of Helsinki). No subject had a history of psychiatric disorders, serious medical illness, or drug abuse in the last 12 months. Subjects had a mean age of $28.1 \pm 10.7$ years, mean verbal NART IQ of $114.8 \pm 6.8$ (Nelson, 1982), body mass index of $24.5 \pm 3.4 \mathrm{~kg} / \mathrm{m}^{2}$, and had been in formal education an average of $15 \pm 3$ years.

Data from one (male) subject was excluded from analysis as assay of plasma levels of ketamine indicated that the level of drug at time of testing was below detectable levels.

\section{Procedure}

The study used a double-blind, placebo-controlled, randomized, within-subjects design. Subjects attended on two occasions receiving a different infusion on each (saline and $100 \mathrm{ng} / \mathrm{ml}$ plasma ketamine). Visits were at least 7 days apart and order of infusion was counterbalanced across subjects.

Bilateral intravenous catheters were inserted into subjects' forearms, one for ketamine infusion, the other for serial blood sampling to assay plasma ketamine levels. Racemic ketamine ( $1 \mathrm{mg} / \mathrm{ml}$ solution) was administered by bolus and continuous infusion using a computerized pump (Graseby 3500, Graseby Medical Ltd, UK). The pump was programmed (Anaetech Ltd, UK) to infuse ketamine continuously at varying doses in order to achieve constant estimated target plasma concentrations of $100 \mathrm{ng} / \mathrm{ml}$, using pharmacokinetic parameters of a three-compartment model (Domino et al, 1982).

\section{Subjective Rating Scales and Clinical Assessments}

During the infusion, subjects were interviewed clinically using an abbreviated form of the Present State Examination, 9th Edition (Wing et al, 1974) designed to focus on symptoms relevant to ketamine's likely effects, and abbreviated to limit the duration of drug exposure.

\section{Reality Monitoring Tasks}

A total of 250 words were selected from the MRC psycholinguistic database (www.psy.uwa.edu.au/mrcdatabase/uwa_mrchtm). In all, 125 words were used on each of the subjects' two visits, randomized across subjects and drug treatment. During each visit, subjects underwent a single study test cycle. 
Study phase. At study, subjects were presented with 100 words. At test, they were presented with the same words plus 25 new words (lures). At study, for each word, a deep (semantic associative) or a shallow (stem completion) encoding task was carried out, and each task carried out on half of the occasions by the experimenter and half of the occasions by the subject themselves. Thus, there were four experimental conditions:

a. Deep encoding - internally generated. An instruction screen appeared $1 \mathrm{~s}$ prior to the presentation of the stimulus word. This consisted of two instructions: the first ('SUBJECT') indicated that the subject was to generate the word, responding aloud. A second instruction ('ASSOCIATE'), presented simultaneously, indicated that they should generate a new word on the basis of a semantic relationship with the word subsequently presented. For example, given the presented word, 'COMPANION', the subject could generate the word 'FRIEND'

b. Shallow encoding - internally generated. The on-screen instruction ('SUBJECT') appeared. In this condition, the task instruction was 'COMPLETE'. Subjects were required to generate a word beginning with the same three letters but grammatically unrelated to the presented word (eg for the presented word: 'PRETEND'; an accepted response would be 'PREFACE', but not 'PRETENDING' or 'PRETENDER').

c. Deep encoding - externally generated. The instruction screen informed the subject that experimenter would provide the response (the word 'EXPERIMENTER' appeared on screen). The second instruction, ('ASSOCIATE') indicated that the subject simply read the subsequent word and listened to the experimenter generate a new word based on a semantic association with the presented word. Words provided by the experimenter were predetermined from a list derived from the MRC psycholinguistic database, and were matched for frequency and number of syllables.

d. Shallow encoding - externally generated. The instruction screen informed the subject that the EXPERIMENTER would perform the task (COMPLETE). The subject observed the presented word and heard the experimenter's response.

Test phase. At study, subjects were instructed that they would later be tested on memory for the words that were presented and for the two sources (task type (associate/ complete) and agency (generated by experimenter/subject)). The test phase was presented $1 \mathrm{~h}$ later. During the test phase, subjects were presented with the 100 words presented during the study phase, and an additional 25 new items. They were required to make a recognition judgement for each item. For words that were rated as previously presented ('old'), subjects tried to recall the task carried out previously on that word (stem completion or semantic association condition) and then the agent who had carried it out (experimenter or subject). Cued recall was also tested by asking the subjects if they could produce the word that was generated by the completion/association. The cued recall task therefore involved the newly generated words, whereas the recognition task involved the presented items.

Thus, subjects were exposed, across the two visits, to a total of 250 words, divided into 10 sets of 25 words (semantic association and stem completion for both external and internal word generation (as described below) and lures on each of two visits) matched for frequency of occurrence in written English (Francis and Kucera, 1982). Words were counterbalanced across foil and targets, as well as across tasks and drug dosage. Words were presented on a computer screen, using DMDX (Forster and Forster, (www.u.arizona.edu/ jforster/dmdx/official.htm)).

\section{Statistical Analysis}

(i) Multinomial modelling. Data analysis aimed to differentiate between key features of the recognition and source memory tasks, taking into account the frequently competing effects of item detection, source memory and guessing biases (Batchelder and Riefer, 1990; Bayen et al, 1996). In order to obtain separate and independent parameter estimates for these critical features, the data were analyzed using a Two-High Threshold multinomial model (Batchelder and Riefer, 1990) via maximum likelihood estimation for item detection, source discrimination and response bias. Multinomial models have previously been successfully applied to source memory data across a range of cognitive paradigms and pathological conditions involving memory deficits (Batchelder et al, 1997; Keefe et al, 2002; Simons et al, 2002), and we have previously used this technique to examine item and source memory deficits following exposure to ketamine (Honey et al, in press).

The multinomial model was implemented using GPT software (X Hu, http://irvin.psyc.memphis.edu/xhuoffice/ gpt/index.htm), and the comparison of individual parameter estimates across treatments was performed using spread-sheet-based algorithms (Dodson et al, 1998).

For each condition, there were four possible responses to each of the three possible sources (associate/complete/new condition: 'associate', 'complete', 'new' or 'don't know'; internal/external/new condition: 'internal', 'external', 'new' or 'don't know'). Response frequencies were recorded for each of these 12 cells under placebo and ketamine conditions, and a model was constructed to account for item recognition and source monitoring, with independent assessment of guessing bias. In order to reduce the complexity of the data, and also to achieve model identifability, two multinomial models were constructed separately for agency and depth, involving parameterization of item recognition (parameters $D_{n}$ ), source discrimination (parameter $d_{1}$ ) and source guesses (parameters $g_{n}$ ). These parameters are further detailed in Figure 1. The multinomial analysis of internal $v s$ external generation combined items from the stem completion and semantic association tasks. Similarly, the model for completion $v s$ association combined internally and externally generated items.

Multinomial models were constructed on the basis of goodness-of-fit of the model, indicated by the log-likelihood ratio statistic, $G^{2}$ (Batchelder and Riefer, 1990; Riefer and Batchelder, 1988), and parsimony, defined as the least number of parameters required to achieve model goodness. 
On this basis, separate models were constructed for response frequencies for associate/complete and internal/ external stimuli, and each was tested under both ketamine and placebo infusion. Mean parameter estimates and SD were based on 100 simulations using data resampling techniques. See Figure 1 for details of model specification.
In a supplementary analysis, the data were also evaluated using standard response measures, $\operatorname{Pr}$ (discrimination) and $\mathrm{Br}$ (response bias) derived from signal detection theory. However, these measures are largely insensitive to guessing biases, and no effects of drug were detected using these measures. These analyses are therefore not reported further.

a Internal vs External Generation

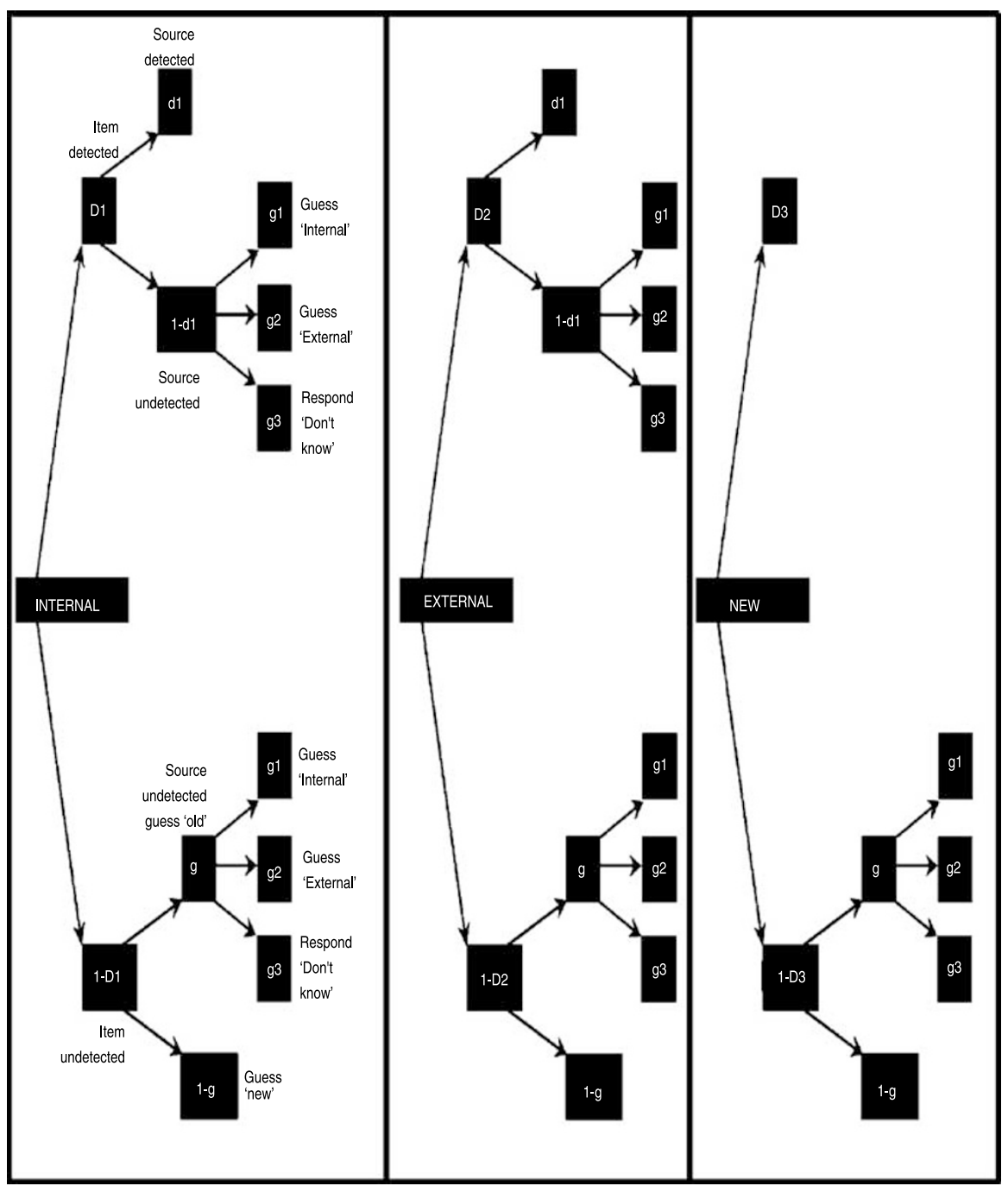

Figure I Multinomial model. (a) Internal vs External Word Generation Model: model estimation provided separate parameter estimates for correct detection of items generated by Subject or Experimenter or New items (parameters $D_{1}, D_{2}$, and $D_{3}$, respectively). Correct source discrimination was modelled by a single parameters $d_{1}$. It was not possible to identify a model which permitted separate parameter estimates for internal vs external source recognition, empirically demonstrated by model goodness indices; this was therefore represented by a single parameter, $d_{1}$. We were therefore not able in this study to determine whether ketamine differentially affects source memory for internally- compared to externally-generated items. However, the parameter that was arguably more central to our hypothesis was subjects' guessing bias, which occurs when source information is not recalled, and subjects therefore engage strategies to source these items. This parameter allowed differentiation between internal and external guessing biases. The observation that under ketamine subjects are less likely to externalize source judgements and do not show an increased tendency to internalize guessing does not support a deficit in reality monitoring. Guessing parameters for item (parameter g) and source (parameter $g_{1}, g_{2}$, and $g_{3}$ ) memory were also modelled. Source memory guessing biases were allowed to vary according to biases towards internal $\left(g_{1}\right)$, external $\left(g_{2}\right)$, or don't know $\left(g_{3}\right)$ responses. The parameterization of the 'don't know' response for agency source $\left(g_{3}\right)$ means that while this response was included in the model, confidence intervals are not available for this item, since it is calculated on the basis of the probability of $I-g_{1}-g_{2}=g_{3}$, and between-group comparisons are therefore not reported. (b) Semantic Association vs Word Stem Completion Model: separate parameter estimates were incorporated for correct detection of items for the Associate and Completion conditions. We assumed that correct detection for Completion items would be similar to that for new items (this was empirically supported by significantly reduced model fit indices for models incorporating separate parameter estimates for detection of new and completion items), and so were modelled as a combined parameter, $D_{1}$. Correct source discrimination was modelled by parameters $d_{1}$. Guessing parameters for source memory were also separately modelled, and allowed to vary according to whether the subject guessed the source $\left(g_{1}\right)$ or responded don't know ( $\left.g_{2}\right)$. 
b

Association vs Completion

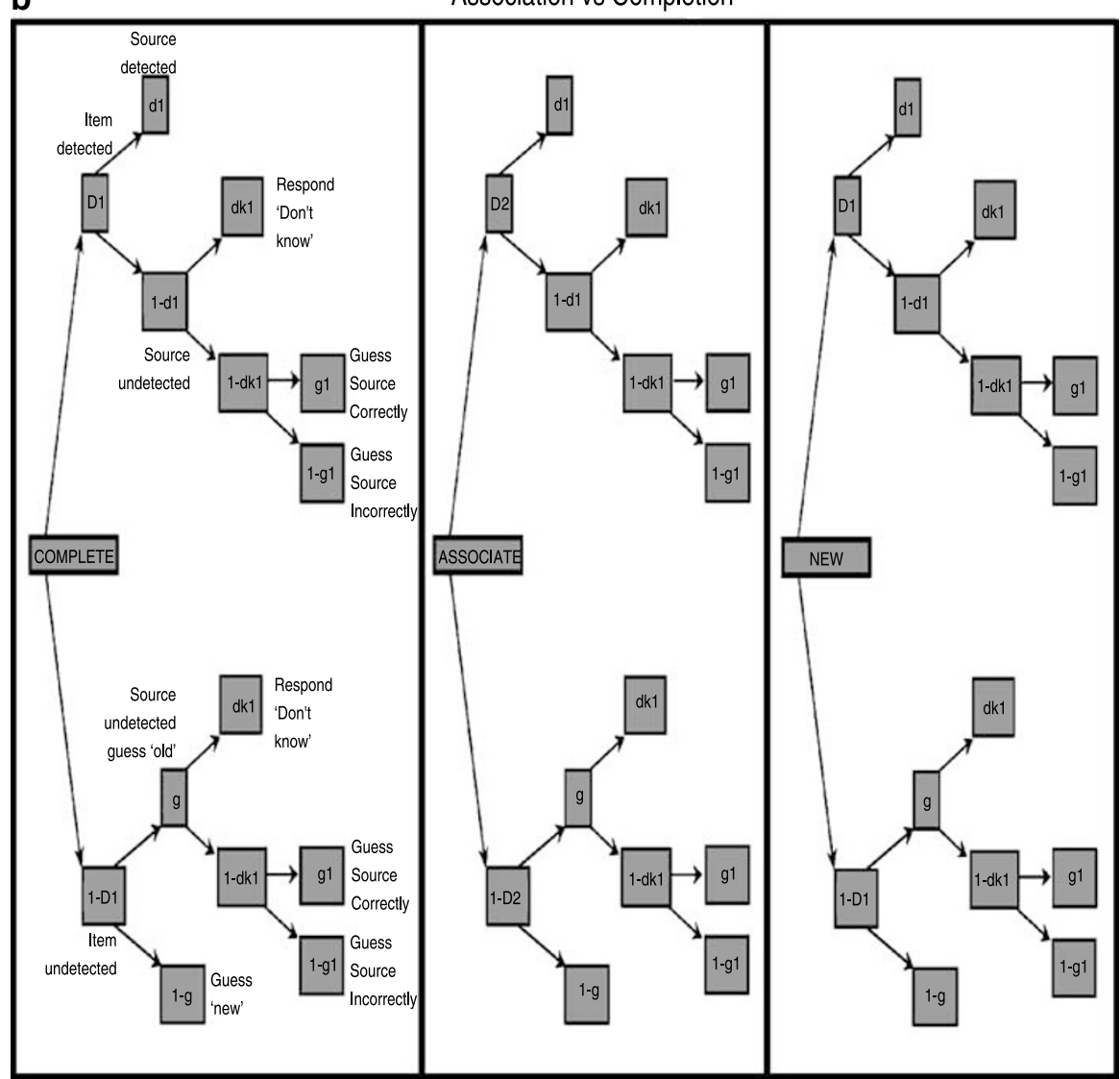

Figure I Continued.

(ii) Word recall. The number of generated words recalled at the test phase was also examined using the above factorial $2 \times 2 \times 2$ repeated measures ANOVA model.

\section{RESULTS}

\section{Plasma Levels}

Observed ketamine plasma levels were in accordance with those predicted by the model $(93.4 \pm 29.4 \mathrm{ng} / \mathrm{ml})$.

\section{Subjective Ratings and Clinical Assessments}

The ratings from the PSE- 9 are presented in Table 1. As observed in our previous studies using these doses (Honey et al, 2003, 2004, 2005; Honey et al, in press), symptoms elicited were minimal and largely nonspecific. The dose was chosen in anticipation that this would be the case, allowing interpretation of cognitive effects in the relative absence of potentially confounding clinical effects of the drug.

\section{Verbal Responses to Encoding Task}

Subjects were able to produce an appropriate verbal response for the stem completion task (mean number of trials to which subjects produced a valid response: $94.6 \%$ under placebo and $93.6 \%$ under ketamine) and the semantic association task (subjects responded on $98.3 \%$ of trials for both placebo and ketamine). There was no effect of drug or drug $\times$ task interaction on the number of responses produced $(p>0.05)$.

\section{Multinomial Modelling Parameter Estimates}

Internal vs external word generation. Recognition: Parameter estimates and goodness of fit indices for the multinomial model are presented in Figure 2. As predicted, comparison of the parameter estimates for the internal/ external word generation model indicates that under both treatment conditions, item recognition was higher for internally generated (placebo: $D_{1}=0.74 \pm 0.02$; ketamine: $D_{1}=0.73 \pm 0.015$ ) than externally generated words (placebo: $D_{2}=0.49 \pm 0.03$; ketamine: $D_{2}=0.46 \pm 0.02$ ), indicating the expected effect that self-generation produced deeper encoding.

Source: The source discrimination score was not reduced under ketamine. However, when source information was not recalled, leading to a guess response, ketamine treatment was associated with a reduced tendency to guess the origin as external. This was indicated by the nonoverlapping confidence intervals of the parameter estimates between treatments for the guessing parameter, $g_{2}$ (placebo: $0.71 \pm 0.03$; ketamine: $0.61 \pm 0.03$ ) (see Figure 2). Betweentreatment differences for the $g_{2}$ parameter estimates were confirmed by a nested model comparison, fitting the model under the constraint that the parameter estimate for the ketamine-treated conditions were equal to the placebo 
condition, and comparing this to the model fit when both conditions were allowed to take different values between conditions. The $g_{2}$ parameter for the ketamine treatment was significantly different from placebo $\left(G^{2}(1)=6.226\right.$, $p=0.013)$.

Semantic association vs stem completion. Model goodness indices showed that separate modelling of source parameters for associate and completion tasks was inappropriate. A single parameter, $d_{1}$, was therefore used to represent source memory for both task types. Similarly, guessing

Table I PSE Clinical Ratings: Number of Subjects Exhibiting Symptoms Rated Using the PSE

\begin{tabular}{lcc}
\hline Subscale & Placebo & Ketamine \\
\hline Tiredness & 2 & 7 \\
Subjective feeling 'nervous tension' & 1 & 2 \\
Autonomic anxiety & 0 & 2 \\
Subjectively inefficient thinking & 3 & 12 \\
Poor concentration & 3 & 11 \\
Depressed mood & - & 3 \\
Simple ideas of reference & - & 1 \\
Expansive mood & 3 & 3 \\
Subjective ideomotor pressure & - & - \\
Derealization & - & - \\
Depersonalitation & - & 1 \\
Delusional mood & - & 0 \\
Heightened perception & - & - \\
Dulled perception & - & 3 \\
Changed perception & - & - \\
Changed perception time, deja vu & - & - \\
Auditory hallucinations & - & - \\
Visual hallucinations & - & - \\
Olfactory hallucinations & - & - \\
Delusions of reference & & - \\
\hline Subcts showed & -
\end{tabular}

Subjects showed an increase in symptoms under ketamine. Most of the subjective symptoms were either nonspecific (eg tiredness, inefficient thinking, poor concentration), or typical ketamine effects (eg heightened perception, changed perception). No subject experienced hallucinations. biases were modelled using a single parameter, $g_{1}$. Parameter estimates and goodness of fit indices for the multinomial model are presented in Figure 3.

Recognition: As expected, comparison of the parameter estimates for the associate/complete model indicates that under both treatment conditions, recognition memory was higher for items for which subjects had generated a semantic association (placebo: $D_{2}=0.75 \pm 0.01$; ketamine: $\left.D_{2}=0.7 \pm 0.01\right)$ compared to word-stem completion (placebo: $D_{1}=0.4 \pm 0.015$; ketamine: $D_{1}=0.42 \pm 0.01$ ), in accordance with the mnemonic advantage of deep encoding (Craik and Lockhart, 1972). Nonoverlapping confidence intervals of the parameter estimates for recognition of 'Associate' words (parameter $D_{2}$ ) indicated that item recognition was significantly reduced for these items under ketamine, but not for the word-stem completion items $\left(D_{1}\right)$ (see Figure 3). Between-treatment differences for the $D_{2}$ parameter estimates were confirmed by a nested model comparison, as described above: the $D_{2}$ parameter for the ketamine treatment was significantly different from placebo $\left(G^{2}(1)=3.979, p=0.046\right)$.

Source: Under ketamine treatment, subjects also showed an increased tendency to respond, 'don't know' (parameter $d k_{1}$ ) to the source judgement (placebo: $d k_{1}=0.16[ \pm 0.02]$; ketamine: $d k_{1}=0.25[ \pm 0.025]$ ). Nested model comparison confirmed the between-treatment difference for this parameter $\left(G^{2}(1)=5.983, p=0.014\right)$.

\section{Word Recall}

Mean numbers of words recalled under each condition are detailed in Figure 4. A main effect of both depth and agency was observed on the number of words subjects recalled: subjects recalled more words from the word association compared to the stem completion condition $(p<0.0001)$ and more words which were internally compared to externally generated $(p<0.0001)$. A drug $\times$ depth interaction was also observed: the increased word recall associated with deep encoding was reduced under ketamine compared to placebo (see Figure 4).

\section{Summary of Findings}

Mnemonic advantage for deep encoding strategies was found for both semantic association (compared to word-

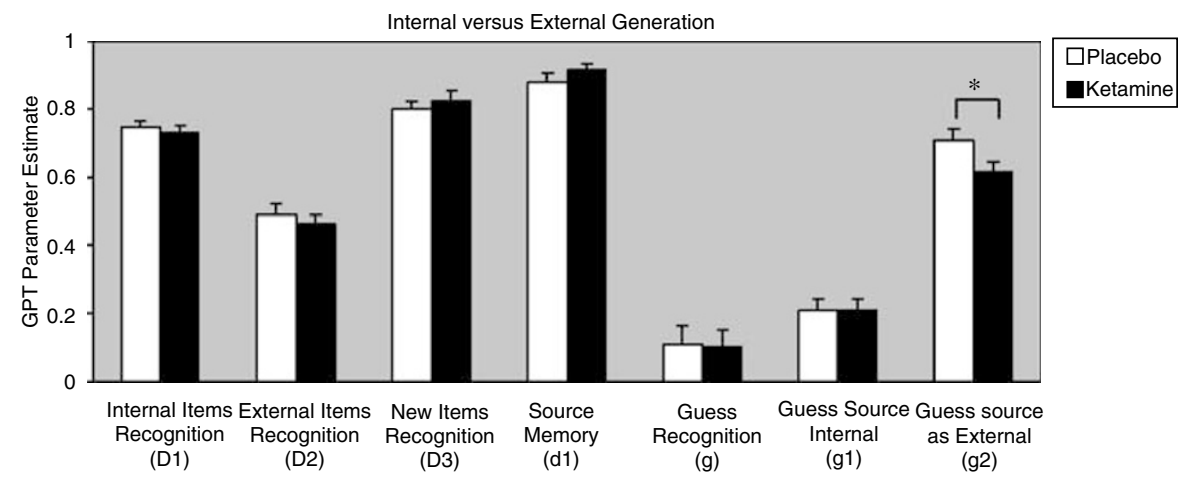

Figure 2 Multinomial modelling parameter estimates for the internal and externally generated items. Asterisk indicates nonoverlapping confidence intervals of the parameter estimates for placebo and ketamine. 


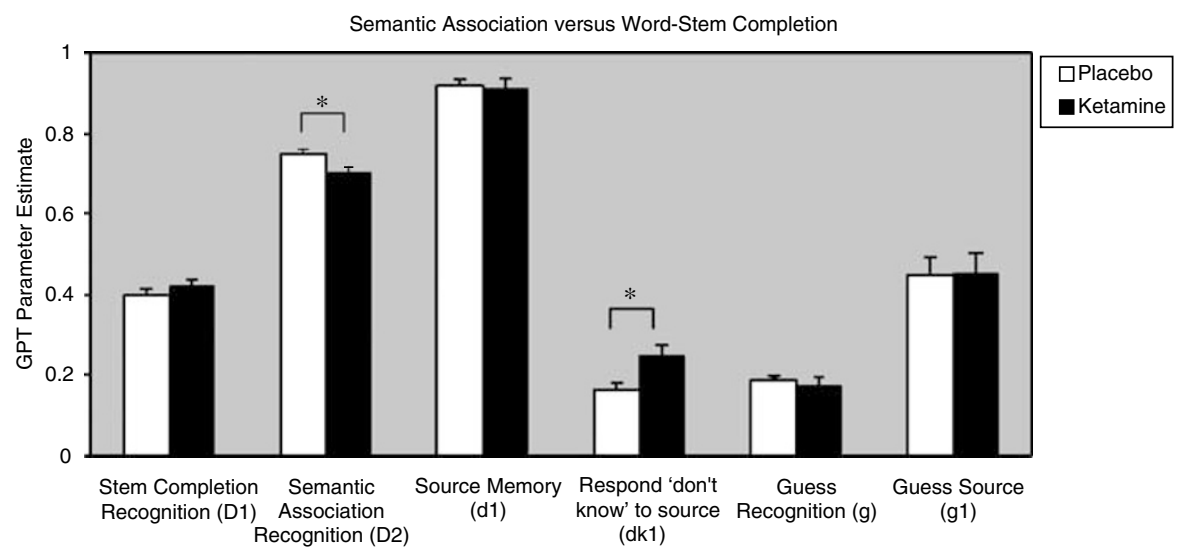

Figure 3 Multinomial modelling parameter estimates for the semantic association and stem-completion items. Asterisks indicates nonoverlapping confidence intervals of the parameter estimates for placebo and ketamine.

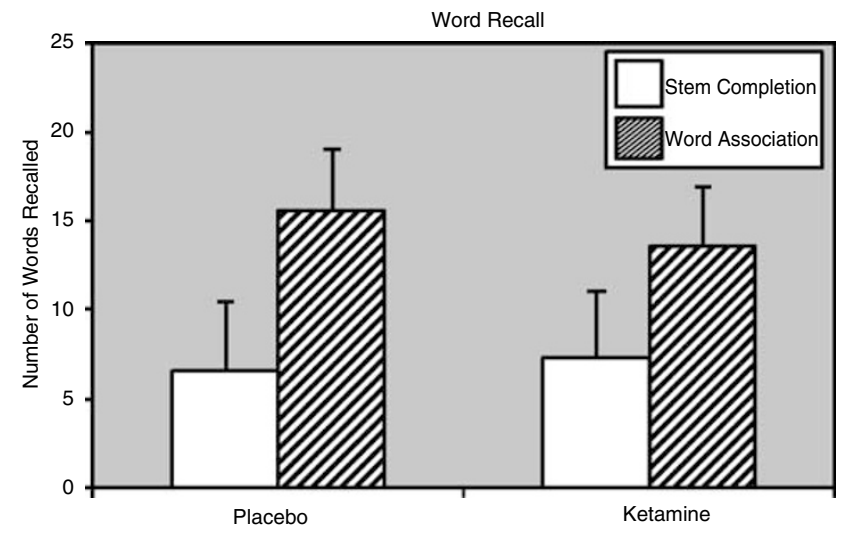

Figure 4 Number of generated words recalled under placebo and ketamine.

stem completion) and internal (compared to external) word generation. Three key effects of ketamine were observed:

(i) With respect to the recognition component of our task, ketamine was associated with a deficit following deeper encoding, as observed in our previous work (Honey et al, in press). There was no measurable effect, however, on the relative advantage to subsequent recognition for internal over external generation.

(ii) Source memory was disrupted under ketamine, manifest as an increased tendency to provide a 'don't know' response for associate/complete source judgements. Guessing parameters for internal/external source memory were affected by the drug. There was, contrary to prediction, a reduced tendency to guess the source as external (ie to attribute source to the experimenter) under ketamine when source information was not recalled.

(iii) There was a reduction under ketamine in the relative advantage to cued recall of deeply (associative) over shallowly (completion) encoded words.

\section{DISCUSSION}

We previously showed that the impact of ketamine upon recognition is dependent upon the level of processing that occurs at encoding, an observation that we replicated in the current study. With respect to source memory for encoding context, we previously showed that low-dose ketamine does not have an impact upon source discrimination measures but does change subjects' response biases. The current study provides further evidence for this and additionally suggests that the encoding-specific effect of ketamine may be observed when cued recall is used as a measure of episodic memory. Thus, our findings replicate our previous observations (Honey et al, in press). Critically, in the current study, we have extended our experimental design to include source memory judgement for agency, a requirement that has relevance to the validity of ketamine as a model for schizophrenia. We show that, when subjects are required to decide whether they or an external agent performed an operation on a word, performance appears unimpaired but, under circumstances in which source information is not recalled, they are less inclined to attribute it to the external agent. Below we consider in greater detail the nature of the disruptions observed under ketamine in terms of the three memory tests used: recognition, source memory and cued recall. We will discuss the potential limitations of the study, and the implications of these findings for the ketamine model of schizophrenia.

\section{Ketamine and Recognition Memory}

Ketamine clearly has a deleterious effect on recognition memory (Ghoneim et al, 1985; Harris et al, 1975; Hetem et al, 2000; Krystal et al, 1994; Malhotra et al, 1996; Newcomer et al, 1999; Radant et al, 1998). Our previous results suggest that this is more pronounced when recognition is based upon recollection rather than familiarity (Honey et al, in press), a view supported by the current data. Item detection was significantly impaired for words in which the encoding task emphasized semantic association rather than stem completion. Orientation towards semantic attributes produces a deeper level of 
encoding and a consequently higher level of recollection than orientation towards orthography, which would be found in the stem-completion task. Thus, as we have argued previously, the impact of ketamine on the semanticassociation task but not upon the completion task indicates that recollection-based as opposed to familiarity-based retrieval is vulnerable to the drug.

One might speculate that any encoding task that produces an improvement in subsequent retrieval shows vulnerability to the effects of ketamine. Thus, it could be that the apparent depth specificity of our observed effects is, in fact, produced by floor effects in the shallower encoding conditions such that the deleterious effects of the drug only emerge when better encoding tasks are used. Two observations militate against this argument. First, in our previous study, we showed that, when an even deeper encoding task (a pleasant/unpleasant decision) is introduced, memory is relatively invulnerable to the impact of ketamine. That is, we showed that the deepest and shallowest encoding levels are not measurably impaired while an intermediate level of encoding is (Honey et al, in press). Furthermore, in the current study, higher levels of encoding were produced not just by task manipulation but by agency manipulation, that is, who was actually doing the task. The improvement in recognition memory associated with internally generated responses was not attenuated by ketamine. Thus, the current study is consistent with our previous assertion that the impact of ketamine is not linearly related to the depth of encoding. Rather it may be that ketamine produces an impairment only insofar as encoding is not rendered invulnerable by strong internal representations produced at encoding. Thus, in our original study, the task that emphasizes internal representations of studied items (the pleasant/unpleasant decision) retained its encoding advantage under ketamine and, in the current study, the relative advantage for internal (self) over external (experimenter) processing was also retained.

\section{Ketamine and Source Memory}

When exploring source memory, simple discrimination and conservatism/liberality indices ( $\mathrm{Pr}$ and $\mathrm{Br}$ used in signal detection theory) fail to characterize fully any changes that may occur in response biases, strategy, and guessing. A fuller analysis, using multinomial modelling, enables us to estimate the effects of these aspects of performance and, critically, the impact that the drug may exert. For example, multinomial modelling is required to elucidate the it had to be you' effect (Johnson and Raye, 1981): a strategy employed when source information is not recalled, leading to the tendency to guess the source as being external on the basis that internally-generated items are more readily recognized. In the current study, both on and off drug, subjects, when guessing source were more likely to guess them as external than internal (see Figure 2). This tendency, however, was significantly attenuated by the drug: subjects on sub-psychotic doses of ketamine showed a reduced tendency to guess the source as external. This is an intriguing finding. One explanation is that subjects on ketamine are simply less likely to adopt a useful guessing strategy. However, this seems unlikely since we have previously observed that, when source memory is impo- verished by ketamine, subjects rely on this strategy to a greater extent than under placebo (Honey et al, in press).

Whatever the explanation for this change in strategy under the drug, this significantly decreased tendency to externalize is clearly in contrast to deficits reported in patients with schizophrenia. In such patients, the suggestion is that a core deficit lies in an increased tendency to externalize. Such a failure has been posited as a basis for delusions and hallucinations. Using multinomial modelling, Keefe et al (1999) found that source monitoring deficits are not limited to agency of action, but were also impaired when discriminating between words from two external sources, two internal sources, and one internal and one external source. However, patients with symptoms of thought insertion, voices arguing/commenting, and delusions of control were distinguished from other patients on the basis of a change in ability to discriminate between items they had imagined and items produced by the experimenter. They showed an increased guessing bias towards external generation when source information was not recalled.

The presence of a very different agency source disruption under ketamine at the dose used here may be taken as evidence against ketamine's validity as a model for schizophrenia. However, this would be premature since it is, perhaps, compatible with the particular psychopathological profile associated with ketamine. Complex visual hallucinations have been reported at high doses of ketamine (Vollenweider et al, 1997), however, such phenomena are relatively uncommon in schizophrenia. Auditory hallucinations, which are more frequently associated with the clinical presentation of the illness, are not typically elicited by ketamine. 'Auditory illusory experiences' (hyperacusis) have occasionally been reported (Abel et al, 2003; Duncan et al, 2001; Krystal et al, 1994, 1998; Lahti et al, 2001), however, these appear to be qualitatively different from true hallucinations, and in particular do not seem to involve distortions of agency. The precise effect of ketamine on delusional behavior is somewhat obscured by the method of reporting BPRS factor scores, which is frequently employed in preference to reporting individual items. The positive symptom factor, to which delusional behavior would contribute, is derived from four key items of the BPRS: conceptual disorganization, hallucinatory behavior, suspiciousness, and unusual thought content. Few studies have therefore reported the nature or content of delusional ideation associated with ketamine. Where further detail is available, delusions of reference and paranoia have been reported (Krystal et al, 1994, 1998). Bowdle et al (1998) reported dose-dependent increases in subjective ratings of reference ('I had the idea that events, objects or other people had particular meaning that was specific for me') and suspiciousness ('I had suspicious ideas or the belief that others were against me').

If it is the case that ketamine at subpsychotic doses produces cognitive deficits that might more readily explain paranoia and delusions of reference then it is perhaps unsurprising that our findings do not reflect the source monitoring deficits shown by people experiencing passivity phenomena and auditory hallucinations. Rather, they may be consistent with the specific pattern of effects more commonly produced by the drug: delusions of reference and 
suspiciousness. It appears that the effect of ketamine is not to produce an inappropriate externalization. Perhaps the symptoms that it does produce reflect quite the opposite. Indeed, the reduced tendency to guess that items were externally, rather than internally, generated, and the observation above that encoding tasks in which internal representations may be elaborated and strengthened are relatively invulnerable to the drug's amnestic effects, all suggest that ketamine may reinforce internal representations. We might speculate that this may contribute to the particular form of delusional ideation observed at higher doses. In short, ketamine may mimic some aspects of the psychopathology of schizophrenia but not others.

Ultimately, it will be critical to identify associations between disruption of source attribution and symptomatic expression at higher doses in order to further probe the relationship between the cognitive and psychotogenic effects of ketamine. It is possible that the effects of ketamine, typically observed to be more subtle than those seen in schizophrenia, may more appropriately model the prodromal phase of the illness, or deficits seen in high-risk subclinical populations, such as familial relatives of patients with psychosis.

\section{Ketamine and Cued Retrieval}

The study design offered one further opportunity to determine the effects of ketamine on memory function. Following recognition and source judgements, subjects attempted to recall the word that had been generated (either by themselves or the experimenter) in response to the stimulus word. Subjects recalled significantly fewer generated words under ketamine for the deep, but not the shallow, encoding task. While we must take into account that the nature of cueing is different following the two types of encoding, these findings provide further evidence that the disruption of episodic memory following ketamine treatment results from a disruption of the advantage of deeper encoding processes. This is consistent with a number of previous studies in patients with schizophrenia which have demonstrated a specific disruption of autonoetic awareness (recollection of contextual information of the episodic-encoding event), with relative sparing of noetic awareness (familiarity-based recognition) indicated using source memory tasks (Brebion et al, 2002; Keefe et al, 2002; Moritz et al, 2003; Morrison and Haddock, 1997; Vinogradov et al, 1997).

\section{Caveats and Limitations}

An important initial consideration in the interpretation of these data is that our findings of impaired recognition memory following deep encoding and the reduced tendency to externalize source attribution under ketamine were observed in the context of minimal psychotomimetic effects of the drug. Our aim in this study was to explore the cognitive effects of the drug at low, subpsychotic doses at which the confounding effects of psychotic phenomena could be minimized. However, it is important to consider the possibility that, in choosing a dose which would not produce psychotic effects, we might be missing a change in externalization bias which would occur at a higher dose.
However, this would not account for the fact that we did observe a measurable change in such bias, but in the opposite direction to that which might be predicted. It could be argued that cognitive effects such as the externalization bias of source memory may be state related, that is, integrally related to the psychotic phenomenon, and therefore only observed in conjunction with symptoms such as hallucinations. According to this interpretation, the absence of these symptoms would be sufficient explanation for the lack of an observed cognitive effect. However, externalization of internal generated source information has been observed in several previous studies in healthy volunteers who do not experience hallucinations, but who score highly on psychometric scales used to identify hallucination-prone subjects in nonclinical populations (Bentall and Slade, 1985; Laroi et al, 2004; Rankin and O'Carroll, 1995) suggesting that such a bias is unlikely to be state related. We should also consider the possibility that the externalization bias changes qualitatively with increasing dose of ketamine, being reduced at lower doses and increased at higher doses. Relatively few studies have examined more than a single dose within- or between subjects; however, of those which have, none reported such a qualitative change with changing doses (Morgan et al, 2004a, b; Newcomer et al, 1999; Passie et al, 2003, 2005). Specifically designed studies would be required to explore this possibility that dose-effect relationships are nonlinear.

There are also certain limitations to our model which must be considered in the interpretation of these data. The findings should be regarded cautiously, since the sample size, while comparable to a number of previous related studies, is relatively low. A second consideration concerns the parameters of the multinomial model. It is customary to parameterize source biases separately following successful and unsuccessful item recognition (Batchelder and Riefer, 1990). However, in modelling the current data, there were insufficient degrees of freedom to allow this in the model. In order to have an identifiable model, we therefore accepted the limitation of modelling source guesses following item detection and nondetection as a single entity, since the goodness of fit of this model was significant, and was replicated in both the placebo and ketamine datasets. This potentially limits our interpretation of the drug effect on parameter $g_{2}$, since it combines source attributions following both successful and unsuccessful item recognition, and further, these may have been differentially affected by ketamine, as in schizophrenia (Keefe et al, 1999, 2002). However, if we make the assumption that source guessing biases will not be differentiated by ketamine (or schizophrenia) for items that are undetected, then one would predict that the net effect of combining source bias for detected and undetected items should still reflect an increased tendency to externalize source attributions if ketamine leads to inappropriate externalization of the source of detected items, as in schizophrenia. The current study demonstrates that under ketamine, subjects are less likely, relative to placebo, to externalize source attributions for agency, however a more precise analysis, particularly in reference to schizophrenia, would require the dissociation of these parameters.

In conclusion, the current study has replicated previous findings that ketamine disrupts mnemonic processes 
associated with deep encoding, processes which facilitate subsequent recollection of contextual detail. The observed disruption of source memory for agency was unpredicted, but suggests that positive symptoms theoretically associated with inappropriate source externalization (eg auditory hallucinations, delusions of control, passivity phenomena, and thought insertion) may be separable from those associated with aberrant source internalization (eg suspiciousness and delusions of reference). Ketamine may provide a useful pharmacological model of the latter but not the former.

\section{ACKNOWLEDGEMENTS}

This work was sponsored by a Wellcome Trust grant awarded to PCF. GDH and PRC were also supported by the Wellcome Trust. We thank the staff of the Wellcome Trust Clinical Research Facility, Addenbrooke's Hospital for clinical support, and also the participants involved in the study.

\section{REFERENCES}

Abel KM, Allin MP, Hemsley DR, Geyer MA (2003). Low dose ketamine increases prepulse inhibition in healthy men. Neuropharmacology 44: 729-737.

Batchelder WH, Chosak-Reiter J, Shankle WR, Dick MB (1997). A multinomial modeling analysis of memory deficits in Alzheimer's disease and vascular dementia. J Gerontol B Psychol Sci Soc Sci 52: 206-215.

Batchelder WH, Riefer DM (1990). Multinomial processing models of source monitoring. Psychol Rev 97: 548-564.

Bayen UJ, Murnane K, Erdfelder E (1996). Source discrimination, item detection, and multinomial models of source monitoring. J Exp Psychol-Learning Memory Cognition 22: 197-215.

Bentall R, Slade P (1985). Reality testing and auditory hallucinations: a signal detection hypothesis. Br J Clin Psychol 24: 159-169.

Bentall RP, Baker GA, Havers S (1991). Reality monitoring and psychotic hallucinations. Br J Clin Psychol 30: 213-222.

Bowdle TA, Radant AD, Cowley DS, Kharasch ED, Strassman RJ, Roy-Byrne PP (1998). Psychedelic effects of ketamine in healthy volunteers: relationship to steady-state plasma concentrations. Anesthesiology 88: 82-88.

Brebion G, Amador X, David A, Malaspina D, Sharif Z, Gorman JM (2000). Positive symptomatology and source-monitoring failure in schizophrenia-an analysis of symptom-specific effects. Psychiatr Res 95: 119-131.

Brebion G, Amador X, Smith MJ, Malaspina D, Sharif Z, Gorman JM (1999). Opposite links of positive and negative symptomatology with memory errors in schizophrenia. Psychiatr Res 88: 15-24.

Brebion G, Gorman JM, Amador X, Malaspina D, Sharif Z (2002). Source monitoring impairments in schizophrenia: characterisation and associations with positive and negative symptomatology. Psychiatr Res 112: 27-39.

Brebion G, Smith MJ, Gorman JM, Amador X (1996). Reality monitoring failure in schizophrenia: the role of selective attention. Schizophr Res 22: 173-180.

Brebion G, Smith MJ, Gorman JM, Amador X (1997). Discrimination accuracy and decision biases in different types of reality monitoring in schizophrenia. J Nerv Ment Dis 185: 247-253.

Craik FIM, Lockhart RS (1972). Levels of processing: a framework for memory research. J Verbal Learning Verbal Behav 11: 671-684.
Danion JM, Rizzo L, Bruant A (1999). Functional mechanisms underlying impaired recognition memory and conscious awareness in patients with schizophrenia. Arch Gen Psychiatr 56: 639-644.

Dodson CS, Prinzmetal W, Shimamura AP (1998). Using Excel to estimate parameters from observed data: an example from source memory data. Behav Res Methods Instrum Comput 30: 517-526.

Domino EF, Zsigmond EK, Domino LE, Domino KE, Kothary SP, Domino SE (1982). Plasma levels of ketamine and two of its metabolites in surgical patients using a gas chromatographic mass fragmentographic assay. Anesth Analg 61: 87-92.

Duncan EJ, Madonick SH, Parwani A, Angrist B, Rajan R, Chakravorty $S$ et al (2001). Clinical and sensorimotor gating effects of ketamine in normals. Neuropsychopharmacology 25: 72-83.

Francis WN, Kucera H (1982). Frequency Analysis of English Usage: Lexicon and Grammar. Houghton Mifflin: Boston.

Frith C (1992). The Cognitive Neuropsychology of Schizophrenia. Lawrence Erlbaum: Hove.

Frith CD (1987). The positive and negative symptoms of schizophrenia reflect impairments in the perception and initiation of action. Psychol Med 17: 631-648.

Frith CD, Done DJ (1989). Experiences of alien control in schizophrenia reflect a disorder in the central monitoring of action. Psychol Med 19: 359-363.

Ghoneim MM, Hinrichs JV, Mewaldt SP, Petersen RC (1985). Ketamine: behavioral effects of subanesthetic doses. J Clin Psychopharmacol 5: 70-77.

Harris JA, Biersner RJ, Edwards D, Bailey LW (1975). Attention, learning, and personality during ketamine emergence: a pilot study. Anesth Analg 54: 169-172.

Harvey PD (1985). Reality monitoring in mania and schizophrenia. The association of thought disorder and performance. J Nerv Ment Dis 173: 67-73.

Hetem LA, Danion JM, Diemunsch P, Brandt C (2000). Effect of a subanesthetic dose of ketamine on memory and conscious awareness in healthy volunteers. Psychopharmacology (Berlin) 152: 283-288.

Honey GD, Honey RA, O'Loughlin C, Sharar SR, Kumaran D, Suckling J et al (2005). Ketamine disrupts frontal and hippocampal contribution to encoding and retrieval of episodic memory: an FMRI study. Cereb Cortex 15: 749-759.

Honey GD, Honey RAE, Sharar SR, Turner DC, Pomarol-Clotet E, Kumaran $\mathrm{D}$ et al (in press). Impairment of specific episodic memory processes by sub-psychotic doses of ketamine: the effects of levels of processing at encoding and of the subsequent retrieval task. Psychopharmacology.

Honey RA, Honey GD, O'Loughlin C, Sharar SR, Kumaran D, Bullmore ET et al (2004). Acute ketamine administration alters the brain responses to executive demands in a verbal working memory task: an FMRI study. Neuropsychopharmacology 29: 1203-1214.

Honey RA, Turner DC, Honey GD, Sharar SR, Kumaran D, Pomarol-Clotet E et al (2003). Subdissociative dose ketamine produces a deficit in manipulation but not maintenance of the contents of working memory. Neuropsychopharmacology 30: 30 .

Johnson MK, Raye CL (1981). Reality monitoring. Psychol Rev 88: 67-85.

Keefe RS, Poe MP, McEvoy JP, Vaughan A (2003). Source monitoring improvement in patients with schizophrenia receiving antipsychotic medications. Psychopharmacology (Berlin) 169: 383-389.

Keefe RSE (1998). The neurobiology of disturbances of the self: autonoetic agnosia in schizophrenia. In: Amador XF, David A (eds). Insight and Psychosis. Oxford University Press: New York. 
Keefe RSE, Arnold MC, Bayen UJ, Harvey PD (1999). Source monitoring deficits in patients with schizophrenia; a multinomial modelling analysis. Psychol Med 29: 903-914.

Keefe RSE, Arnold MC, Bayen UJ, McEvoy JP, Wilson WH (2002). Source-monitoring deficits for self-generated stimuli in schizophrenia: multinomial modeling of data from three sources. Schizophr Res 57: 51-67.

Krystal JH, Karper LP, Bennett A, D'Souza DC, Abi-Dargham A, Morrissey $\mathrm{K}$ et al (1998). Interactive effects of subanesthetic ketamine and subhypnotic lorazepam in humans. Psychopharmacology (Berlin) 135: 213-229.

Krystal JH, Karper LP, Seibyl JP, Freeman GK, Delaney R, Bremner JD et al (1994). Subanesthetic effects of the noncompetitive NMDA antagonist, ketamine, in humans. Psychotomimetic, perceptual, cognitive, and neuroendocrine responses. Arch Gen Psychiatr 51: 199-214.

Lahti AC, Weiler MA, Tamara Michaelidis BA, Parwani A, Tamminga CA (2001). Effects of ketamine in normal and schizophrenic volunteers. Neuropsychopharmacology 25: 455-467.

Laroi F, Van der Linden M, Marczewski P (2004). The effects of emotional salience, cognitive effort and meta-cognitive beliefs on a reality monitoring task in hallucination-prone subjects. Br J Clin Psychol 43(Part 3): 221-233.

Malhotra AK, Pinals DA, Adler CM, Elman I, Clifton A, Pickar D et al (1997). Ketamine-induced exacerbation of psychotic symptoms and cognitive impairment in neuroleptic-free schizophrenics. Neuropsychopharmacology 17: 141-150.

Malhotra AK, Pinals DA, Weingartner H, Sirocco K, Missar CD, Pickar D et al (1996). NMDA receptor function and human cognition: the effects of ketamine in healthy volunteers. Neuropsychopharmacology 14: 301-307.

Morgan CJ, Mofeez A, Brandner B, Bromley L, Curran HV (2004a). Acute effects of ketamine on memory systems and psychotic symptoms in healthy volunteers. Neuropsychopharmacology 29: 208-218.

Morgan CJ, Mofeez A, Brandner B, Bromley L, Curran HV (2004b). Ketamine impairs response inhibition and is positively reinforcing in healthy volunteers: a dose-response study. Psychopharmacology 15: 15.

Moritz S, Woodward TS, Ruff CC (2003). Source monitoring and memory confidence in schizophrenia. Psychol Med 33: 131-139.

Morrison AP, Haddock G (1997). Cognitive factors in source monitoring and auditory hallucinations. Psychol Med 27: 669-679.

Nelson H (1982). National Adult Reading Test. NFER-Nelson Publishing Company Ltd: New York.
Newcomer JW, Farber NB, Jevtovic-Todorovic V, Selke G, Melson AK, Hershey T et al (1999). Ketamine-induced NMDA receptor hypofunction as a model of memory impairment and psychosis. Neuropsychopharmacology 20: 106-118.

Olney JW, Farber NB (1995). Glutamate receptor dysfunction and schizophrenia. Arch Gen Psychiatr 52: 998-1007.

Passie T, Karst M, Borsutzky M, Wiese B, Emrich HM, Scneider U (2003). Effects of different subanaesthetic doses of (S)-ketamine on psychopathology and binoculr depth inversion in man. J Psychopharmacol 17: 51-56.

Passie T, Karst M, Wiese B, Emrich HM, Schneider U (2005). Effects of different subanaesthetic doses of (S)-ketamine on Neuropsychology, Psychopathology and State of Conciousness in Man. Neuropsychobiology 51: 226-233.

Radant AD, Bowdle TA, Cowley DS, Kharasch ED, Roy-Byrne PP (1998). Does ketamine-mediated $N$-methyl-D-aspartate receptor antagonism cause schizophrenia-like oculomotor abnormalities? Neuropsychopharmacology 19: 434-444.

Rankin PM, O'Carroll PJ (1995). Reality discrimination, reality monitoring and disposition towards hallucination. $\mathrm{Br} \mathrm{J}$ Clin Psychol 34(Part 4): 517-528.

Riefer DM, Batchelder WH (1988). Multinomial modeling and the measurement of cognitive-processes. Psychol Rev 95: 318-339.

Simons JS, Verfaellie M, Galton CJ, Miller BL, Hodges JR, Graham KS (2002). Recollection-based memory in frontotemporal dementia: implications for theories of long-term memory. Brain 125: $2523-2536$

Slamecka NJ, Graf P (1978). The generation effect: delineation of a phenomenon. J Exp Psychol: Hum Learning Memory 4: 592-604.

Stirling JD, Hellewell JS, Hewitt J (1997). Verbal memory impairment in schizophrenia: no sparing of short-term recall. Schizophr Res 25: 85-95.

Tamminga CA, Holcomb HH, Gao XM, Lahti AC (1995). Glutamate pharmacology and the treatment of schizophrenia: current status and future directions. Int Clin Psychopharmacol 10(Suppl 3): 29-37.

Vinogradov S, Willis-Shore J, Poole JH, Marten E, Ober BA, Shenaut GK (1997). Clinical and neurocognitive aspects of source monitoring errors in schizophrenia. Am J Psychiatr 154: $1530-1537$

Vollenweider FX, Leenders KL, Scharfetter C, Maguire P, Stadelmann O, Angst J (1997). Positron emission tomography and fluorodeoxyglucose studies of metabolic hyperfrontality and psychopathology in the psilocybin model of psychosis. Neuropsychopharmacology 16: 357-372.

Wing J, Cooper J, Sartorius N (1974). The Measurement and Classification of Psychiatric Symptoms. Cambridge Universtiy Press: Cambridge. 